\title{
Caracterización de seis generaciones de egresados de medicina
}

\author{
Characteristics among six generations of medicine graduates
}

Miguel A. Fernández-Ortega ${ }^{*}$, Efrén R. Ponce-Rosas², Armando Ortiz-Montalvo², Guillermo Fajardo-Ortiz² e Irma Jiménez-Galván²

${ }^{1}$ Facultad de Medicina, Centro de Investigación en Políticas, Población y Salud; ${ }^{2}$ Facultad de Medicina, División de Estudios de Posgrado. Universidad Nacional Autónoma de México. Ciudad de México, México

\section{Resumen}

Antecedentes: Los programas de seguimiento de egresados permiten mejorar los programas y el currículo de la carrera de Medicina, identificando las áreas de oportunidad y haciendo más competitivas a las instituciones educativas. Objetivo: Identificar las características sociodemográficas, formativas, de inserción laboral y de satisfacción, en seis generaciones de egresados de la carrera de Medicina de la Universidad Nacional Autónoma de México (2004-2009). Método: Estudio descriptivo, transversal, retrospectivo y comparativo, con 5295 egresados de la carrera de Medicina que concluyeron entre 2004 y 2009. Se envió un cuestionario por correo electrónico a cada uno de los egresados y se analizaron los resultados mediante técnicas descriptivas e inferenciales. Resultados: Se observó un incremento en el número de años para concluir la carrera y un aumento en el número de materias reprobadas en las últimas tres generaciones. Además, hubo mayor lentitud en la inserción laboral en el mismo periodo, aunque el porcentaje de desempleo general fue muy bajo (2.9\%). Conclusiones: No hay cambios en la caracterización de los egresados respecto a generaciones anteriores; no obstante, será fundamental identificar las causas del incremento en el número de materias reprobadas que está afectando la eficiencia terminal, además de mejorar los programas de investigación epidemiológica y socio-médica.

Palabras Clave: Escuela de medicina. Satisfacción. Currículo. Empleo.

\begin{abstract}
Background: Medical graduates follow-up programs allow the improvement of the curriculum of the Medicine career. Through identifying opportunity areas and strengthening points, institutions become increasingly competitive. Objective: Identify the socio-demographic, formative, labor insertion and satisfaction characteristics among six generations of graduates of the Medical School of the Faculty of Medicine of the National Autonomous University of Mexico (2004-2009). Method: It is a descriptive, cross-sectional, retrospective, comparative study integrating the answers of mailed electronic questionnaires to 5295 graduates who concluded studies between 2004 and 2009. Descriptive and inferential techniques were used for analysis. Results: An upward trend in the number of years to conclude the career was identified and an increase in failed assignments in the last three generations. Noticeable was also that, as time went by, the incorporation of physicians into the labor market was slower. Although the percentage of general unemployment was very low (2.9\%). Conclusions: There are no major changes in the
\end{abstract}

\section{Correspondecia:}

*Miguel A. Fernández-Ortega

Zona Cultural Universitaria, Ciudad Universitaria

Del. Coyoacán

Fecha de recepción: 07-11-2018

Fecha de aceptación: 15-01-2020

E-mail: miguelaf03@live.com

DOI: $10.24875 / C I R U .20000881$
Cir Cir. 2020;88(3):269-276

Contents available at PubMed www.cirugiaycirujanos.com
ajo la licencia CC BY-NC-ND 0009-7411/@ 2020 Academia Mexicana de Cirugía.
(http://creativecommons.org/licenses/by-nc-nd/4.0/). 
characteristics of the Medicine graduates. Although it is necessary to identify the reason for the increased in failed assignments which affects the length of completion. Further improvement in Research and Social Medicine programs are to be considered.

Key Words: Medical school. Satisfaction. Curriculum. Employment.

\section{Introducción}

En México, al igual que en muchos otros países del mundo, se ha incrementado en las últimas dos décadas la tendencia a evaluar y certificar los estándares de calidad de los médicos y de las instituciones de salud, y también los de las instituciones educativas ${ }^{1-5}$.

Las instituciones de educación superior, públicas y privadas, reconocen la enorme responsabilidad que tienen en formar los recursos profesionales que habrán de insertarse en el mercado laboral y mantener a la población sana y productiva. En estos momentos las universidades transitan de los procesos formativos de autocomplacencia a los de gran responsabilidad social y administrativa, que buscan perfeccionar sus procesos de formación incluso a través de evaluaciones de pares y autoevaluaciones, como puede interpretarse a los programas de seguimiento de alumnos y egresados (PSAE) $)^{6-9}$.

Los PSAE con que cuentan muchas de las escuelas y facultades de Medicina han permitido avanzar en el mejoramiento de los programas y del currículo de la carrera, al identificar las áreas de oportunidad y fortaleza, haciendo cada vez más competitivas a las instituciones educativas ${ }^{6-8,10}$.

Por lo anterior, resulta evidente el peso que tienen las universidades en la incorporación de los individuos al mercado sanitario, garantizando no solo el bien de la comunidad, sino también el del médico egresado que habrá de incorporarse al mercado laboral para llevar recursos a sus familias ${ }^{11,12}$.

Es conveniente recordar algunas de las teorías clásicas encaminadas a entender el binomio educación vs. trabajo, que pese al tiempo transcurrido desde su publicación siguen teniendo cierta vigencia, aunque sea de manera parcial, ya que ninguna logra explicar por sí sola la relación entre educación y trabajo. Tal es el caso de la teoría del capital humano de Theodore Schultz, en 1959, que consideraba que el sistema educativo traería consigo una mayor calificación de la fuerza de trabajo y, por consiguiente, una mayor producción y crecimiento económico, mejor remuneración de los trabajadores y mejor distribución del ingreso ${ }^{4,13}$. A esta teoría, Frederick Harbison y Charles Myers $^{13}$ agregaron que la educación media y superior per cápita se encontraba altamente correlacionada con el ingreso per cápita de un país ${ }^{4}$.

Otras teorías relevantes son las de los sociólogos Sewell-Hauser y Blaug-Duncan, las cuales señalan una estrecha relación entre la educación de los padres y los logros educativos de los hijos ${ }^{4,13}$.

Existen otras teorías, como la teoría de la fila de Arrow, que señala que la educación no contribuye directamente al crecimiento económico, sino que sirve como un medio para seleccionar a la gente y asignarles empleos; la teoría de la devaluación de los certificados, que explica el fenómeno relacionado con una creciente necesidad de escolaridad para incorporarse al mercado laboral por parte de los egresados, en relación con los empleados que se van retirando, con una tendencia a una menor remuneración por el mismo trabajo; la teoría del bien posicional, que considera que los salarios se estructuran según la naturaleza de los empleos y no sobre la base de las características del capital humano; el modelo de la segmentación, que al igual que la teoría del bien posicional considera que para la organización del trabajo son más importantes las características organizativas que las del profesional ${ }^{4,5,14}$; y las teorías de la asignación y del conocimiento heterogéneo, descritas por Sattinger (1993) y por Allen y Van Der Valden (2001), que comparten la premisa de que las retribuciones son determinadas por dos factores: el nivel educativo del sujeto y las características del puesto de trabajo (la diferencia entre ambas estriba en la subutilización de conocimientos para desempeñar un puesto de trabajo, que señala la teoría de la asignación, pero no así la del conocimiento heterogéneo) $)^{15}$.

Existen múltiples PSAE que han confirmado mucha de la información descrita en estas y otras teorías. En este estudio se analizan las características sociodemográficas, formativas, de inserción laboral y de satisfacción en seis generaciones de egresados de la carrera de Medicina de la Facultad de Medicina de la Universidad Nacional Autónoma de México (2004-2009).

\section{Método}

El estudio representa la primera fase de análisis de la investigación. Se describen variables descriptivas que caracterizan la muestra estudiada de seis 
Tabla 1. Distribución de egresados encuestados por generación y sexo

\begin{tabular}{|c|c|c|c|c|c|}
\hline \multirow[t]{2}{*}{ Año de egreso } & \multirow[t]{2}{*}{ Frecuencia } & \multirow[t]{2}{*}{ Porcentaje } & \multicolumn{2}{|c|}{ Sexo } & \multirow[t]{2}{*}{ Total } \\
\hline & & & Hombres & Mujeres & \\
\hline 2004 & 147 & 10.3 & 71 & 76 & 147 \\
\hline 2005 & 192 & 13.4 & 78 & 114 & 192 \\
\hline 2006 & 223 & 15.6 & 90 & 133 & 223 \\
\hline 2007 & 267 & 18.6 & 115 & 152 & 267 \\
\hline 2008 & 295 & 20.6 & 107 & 188 & 295 \\
\hline 2009 & 310 & 21.6 & 121 & 189 & 310 \\
\hline Total & 1434 & 100 & $582(40.6 \%)$ & 852 (59.4\%) & 1434 (100\%) \\
\hline
\end{tabular}

generaciones de egresados. Es un estudio descriptivo, transversal, retrospectivo y comparativo, con 5295 egresados de la carrera de Medicina que concluyeron sus estudios entre los años 2004 y 2009. Se elaboró un cuestionario con 62 variables relacionadas con factores sociodemográficos, educativos, de desempeño profesional (laboral) y de satisfacción de los egresados. Se realizó una prueba piloto con un grupo equivalente al estudiado $(n=15)$, y con los resultados de la prueba se realizaron ajustes de semántica y redacción de las preguntas. Se validó en su contenido por ocho profesores de la facultad de Medicina con experiencia docente mayor de 10 años. No se consideró incursionar en la validez de criterio ni de constructo, ya que el estudio es de tipo exploratorio en su etapa inicial. Se elaboró un directorio de egresados de las generaciones 2004 a 2009, con información de los expedientes proporcionada por la Secretaría de Servicios Escolares de la Facultad. Posteriormente, el cuestionario fue enviado por correo electrónico a cada uno de los egresados y se dio un margen de 2 meses para su respuesta; posteriormente se enviaron recordatorios cada 2 semanas hasta completar 8 meses en la compilación de encuestas. Finalmente, a los médicos que no respondieron el cuestionario por vía electrónica se les pidió una cita para realizar la aplicación del cuestionario de modo presencial, lo que llevó aproximadamente 2 meses más.

Se realizó el análisis estadístico mediante técnicas descriptivas e inferenciales utilizando el programa SPSS V22. El proyecto fue aprobado por la Comisión de Investigación y Ética de la División de Investigación de la Facultad, apegándose a las recomendaciones éticas de investigación en educación de la Ethical Standards of American Educational Research Association (2000) y la Declaración de Helsinki (2013) ${ }^{16,17}$.

\section{Resultados}

De los 5295 egresados, solo 1434 aceptaron responder el cuestionario (Tabla 1).

\section{Perfil general}

La distribución por sexo en la muestra total fue del $59.4 \%(n=852)$ mujeres y el $40.6 \%(n=582)$ hombres. El $61.6 \%$ tenían entre 30 y 34 años de edad. Durante la carrera, el $93.2 \%$ fueron solteros, sin hijos el $93.9 \%$. Trabajaban el $14.1 \%$, y de estos el $56.9 \%$ lo hacían entre 8 y 20 horas a la semana y el $13.4 \% 40$ horas en empleos que no tenían relación con el área de la salud. El tiempo de traslado de los alumnos para asistir a sus estudios en la Facultad de Medicina fue entre 90 y 120 minutos en el $35.4 \%$ de los casos.

\section{Antecedentes escolares}

La gran mayoría de los alumnos (97.4\%) no reprobó años durante su instrucción escolar básica, media y superior (primaria, secundaria y bachillerato); el 93.3\% obtuvo un promedio escolar en el bachillerato de entre 8 y 9.9 (escala de 5 a 10). El 67.2\% de los alumnos terminó el plan de estudios de medicina en 6.5 años y el $31.9 \%$ lo hizo entre 1 y 2 años después. El $60 \%$ de los alumnos no reprobaron materias durante la carrera y el $30.8 \%$ reprobaron una a dos materias. Por lo que se refiere al tipo de actividad principal realizada en el Servicio Social, el $73 \%$ lo hizo en clínicas rurales proporcionando atención médica a la población. Del total de la muestra, el $86.2 \%$ realizó algún curso de especialización médica y el $13.8 \%$ no lo hizo. El $16.3 \%$ tiene estudios de maestría y solo el $2.4 \%$ tiene un doctorado. 
La institución de salud más frecuente para realizar la especialidad fue la Secretaría de Salud (Ministerio de Salud), con el 34\%, seguida del Instituto Mexicano del Seguro Social con el $29.7 \%$. Solo el $66.5 \%$ de los médicos lograron ingresar al sistema de residencias médicas (especialidades) en su primer intento, el $11.0 \%$ en el segundo y el $6.1 \%$ en el tercero.

Respecto al tiempo transcurrido entre el término de la carrera y el ingreso a la especialidad médica, el $37.9 \%$ lo logró inmediatamente y el $39.6 \%$ lo hizo entre 1 y 2 años después.

El $51.9 \%$ de los médicos manifestó haber realizado actividades de educación continua entre una y tres ocasiones en el último año, y el $22.6 \%$ no las realizó. Los cursos y los congresos fueron las actividades de educación continua más frecuentes (63\%). El $71.1 \%$ de los entrevistados manifestó tener la certificación vigente de su especialidad médica.

\section{Aspectos laborales}

Al terminar la carrera de Médico Cirujano, el $72.7 \%$ lograron un empleo profesional en menos de 6 meses, el $10.3 \%$ lo hicieron en 6 meses a 1 año, y solo el $2.6 \%$ en 1 a 3 años. Los factores considerados más importantes para lograr la contratación laboral fueron el desempeño profesional (32.8\%), la acreditación del examen de selección de personal $(20.2 \%)$ y la entrevista laboral exitosa (17.3\%).

En el momento del estudio, el $48.7 \%$ tenía un empleo, el $38.5 \%$ dos y solo el $2.9 \%$ se encontraba desempleados. El $44.2 \%$ de los médicos manifestó laborar en instituciones públicas, el $19.9 \%$ en privadas y el $35.9 \%$ en ambas.

El cargo principal ejercido fue como médico especialista clínico (76\%). El $20.3 \%$ obtuvo su primer ascenso laboral en menos de 1 año y el $17 \%$ en 1 a 4 años; el $40.5 \%$ nunca ha tenido un ascenso laboral.

\section{Satisfacción del egresado}

El $96.9 \%$ de los entrevistados opinaron que la formación profesional recibida en la Facultad de Medicina les ha permitido un desempeño favorable; el $56 \%$ se autoevalúan como excelentes y el $43.9 \%$ como buenos. Estos datos sustentan la afirmación de los encuestados, que se consideran médicos exitosos en el $95 \%$ de los casos.

Los elementos formativos más frecuentes que los médicos dicen aplicar en su ejercicio profesional se presentan en la tabla 2.
Tabla 2. Elementos adquiridos en la formación profesional de los egresados

\begin{tabular}{lc}
\hline Elementos formativos & Porcentaje \\
\hline $\begin{array}{l}\text { Referencia oportuna de los pacientes a } \\
\text { especialidades médicas }\end{array}$ & 96.4 \\
$\begin{array}{l}\text { Trabajo en equipo con otros profesionales de la } \\
\text { salud }\end{array}$ & 94.9 \\
Enfoque de prevención de riesgos & 90.2 \\
Atención médica integral & 89.8 \\
$\begin{array}{l}\text { Atención de las necesidades psicológicas y } \\
\text { afectivas de los pacientes }\end{array}$ & 75.2 \\
$\begin{array}{l}\text { Utilidad para comprender la investigación clínica } \\
\text { Utilidad para comprender los protocolos de }\end{array}$ & 70.7 \\
investigación diagnóstico-terapéuticos & 67.5 \\
Relación médico-paciente & 67.3 \\
Responsabilidad profesional & 66.8 \\
Respeto y autonomía del paciente & 59.8 \\
Consentimiento informado & 55.0 \\
Manejo del expediente clínico & 49.7 \\
$\begin{array}{l}\text { Utilidad para comprender los estudios } \\
\text { epidemiológicos } \\
\text { Implicaciones médico-legales }\end{array}$ & 43.3 \\
Utilidad para comprender los protocolos de & 39.6 \\
\hline
\end{tabular}

Los egresados manifestaron que las bases éticas y humanísticas proporcionadas durante el transcurso de la carrera fueron las adecuadas para el ejercicio profesional (tabla 3).

El $98 \%$ de los médicos recomendarían la Facultad de Medicina para realizar la carrera de Medicina. Respecto a la planta académica, el $56.5 \%$ calificaron a los profesores del área básica como buenos y el $41.4 \%$ como excelentes; por lo que se refiere a los profesores del área clínica, el $57.25 \%$ los considera buenos y el $39.8 \%$ excelentes.

El plan de estudios que cursaron lo consideraron bueno el $63.9 \%$ y excelente el $32.6 \%$, y el $83.5 \%$ indica que les facilitó su inserción en el mercado laboral.

Las principales fortalezas de la Facultad fueron el programa académico $(26.4 \%)$, el prestigio $(27.1 \%)$, los profesores (22\%) y los campos clínicos (18.8\%). Sus principales debilidades fueron los trámites administrativos (30.5\%), los conflictos estudiantiles $(23.4 \%)$, el programa académico $(10 \%)$ y el gran número de alumnos en los grupos (8\%). 
Tabla 3. Tendencia en el número de años en que los egresados terminaron la carrera

\begin{tabular}{|c|c|c|c|c|c|c|c|c|c|c|c|c|}
\hline \multirow[t]{3}{*}{ Generación } & \multicolumn{12}{|c|}{ Tiempo para terminar la carrera } \\
\hline & \multicolumn{2}{|c|}{6,5 años } & \multicolumn{2}{|c|}{ 7,5 años } & \multicolumn{2}{|c|}{8,5 años } & \multicolumn{2}{|c|}{9,5 años } & \multicolumn{2}{|c|}{10 o más años } & \multicolumn{2}{|c|}{ Total } \\
\hline & $\mathrm{N}$ & $\%$ & $\mathbf{N}$ & $\%$ & $\mathrm{~N}$ & $\%$ & $\mathbf{N}$ & $\%$ & N & $\%$ & $\mathrm{~N}$ & $\%$ \\
\hline 2004 & 120 & 81.6 & 26 & 17.7 & 1 & 0.7 & 0 & 0 & 0 & 0 & 147 & 100 \\
\hline 2005 & 139 & 72.4 & 47 & 24.5 & 6 & 3.1 & 0 & 0 & 0 & 0 & 192 & 100 \\
\hline 2006 & 157 & 70.4 & 45 & 20.2 & 18 & 8.1 & 3 & 1.3 & 0 & 0 & 223 & 100 \\
\hline 2007 & 176 & 65.9 & 76 & 28.5 & 10 & 3.7 & 4 & 1.5 & 1 & 0.4 & 267 & 100 \\
\hline 2008 & 189 & 64.1 & 88 & 29.8 & 17 & 5.8 & 1 & 0.3 & 0 & 0 & 295 & 100 \\
\hline 2009 & 182 & 58.7 & 91 & 29.4 & 32 & 10.3 & 4 & 1.3 & 1 & 0.3 & 310 & 100 \\
\hline Total & 963 & & 373 & & 84 & & 12 & & 2 & & 1434 & 100 \\
\hline
\end{tabular}

Tabla 4. Materias no acreditadas por los egresados según generación

\begin{tabular}{|c|c|c|c|c|c|c|c|c|c|c|c|c|c|c|c|c|}
\hline \multirow[t]{3}{*}{ Generación } & \multicolumn{16}{|c|}{ Número de materias no acreditadas en la carrera } \\
\hline & \multicolumn{2}{|c|}{ Todas aprobadas } & \multicolumn{2}{|c|}{ Una } & \multicolumn{2}{|c|}{ Dos } & \multicolumn{2}{|c|}{ Tres } & \multicolumn{2}{|c|}{ Cuatro } & \multicolumn{2}{|c|}{ Cinco } & \multicolumn{2}{|c|}{ Seis o más } & \multicolumn{2}{|c|}{ Total } \\
\hline & $\mathbf{N}$ & $\%$ & $\mathbf{N}$ & $\%$ & $\mathbf{N}$ & $\%$ & $\mathrm{~N}$ & $\%$ & $\mathrm{~N}$ & $\%$ & $\mathrm{~N}$ & $\%$ & $\mathbf{N}$ & $\%$ & $\mathrm{~N}$ & $\%$ \\
\hline 2004 & 98 & 66.7 & 33 & 22.4 & 9 & 6.1 & 2 & 1.4 & 2 & 1.4 & 0 & 0 & 3 & 2 & 147 & 100 \\
\hline 2005 & 133 & 69.3 & 24 & 12.5 & 23 & 12 & 7 & 3.6 & 3 & 1.6 & 2 & 1 & 0 & 0 & 192 & 100 \\
\hline 2006 & 138 & 61.9 & 46 & 20.6 & 21 & 9.4 & 6 & 2.7 & 8 & 3.6 & 2 & 0.9 & 2 & 0.9 & 223 & 100 \\
\hline 2007 & 161 & 60.3 & 53 & 19.8 & 32 & 12 & 13 & 4.9 & 7 & 2.6 & 1 & 0.4 & 0 & 0 & 267 & 100 \\
\hline 2008 & 169 & 57.3 & 58 & 19.7 & 39 & 13.2 & 20 & 6.8 & 4 & 1.4 & 2 & 0.7 & 3 & 1 & 295 & 100 \\
\hline 2009 & 162 & 52.3 & 63 & 20.3 & 41 & 13.2 & 21 & 6.8 & 14 & 4.5 & 3 & 1 & 6 & 1.9 & 310 & 100 \\
\hline Total & 861 & & 277 & & 165 & & 69 & & 38 & & 10 & & 14 & & 1434 & 100 \\
\hline
\end{tabular}

\section{Análisis longitudinal de seis generaciones}

En este apartado se describen las tendencias observadas durante el periodo de seguimiento de 2004 a 2009.

De manera consistente y en valores porcentuales promedio, egresaron de la carrera de Medicina un $60 \%$ de mujeres y un $40 \%$ de hombres. El $93 \%$ eran solteros y $\sin$ hijos. Trabajaban el $14 \%$ y lo hacían en más del $60 \%$ entre 8 y 20 horas semanales, siendo en su mayoría empleados (51.7 a 67.4\%).

Los egresados obtuvieron un promedio de ingreso a la carrera de Medicina de 8.9 a 9 en el 27.1 al $35 \%$ de los casos, y un promedio de 9.0 a 9.9 en el 58.1 al $65.6 \%$, según la generación de referencia.

El número de años en que se cursó la carrera muestra un patrón muy claro con tendencia al alza (Tabla 3). Se observa que para 7.5 y 8.5 años, los alumnos han aumentado significativamente a través del periodo considerado (ji al cuadrado de Pearson: 53.29; $p<0.0001$ ).

Los alumnos que suspendieron la carrera al menos 1 año fue muy similar en las seis generaciones, alrededor del $9.7 \%$. Los principales motivos por los que los egresados suspendieron sus estudios fueron razones económicas, presencia de alguna enfermedad y motivos familiares.

La tasa porcentual promedio de aprobación de todas las asignaturas de la carrera para las seis generaciones fue del $60 \%$, de reprobación de una materia el $19.3 \%$ y de dos materias el $11.5 \%$. La diferencia fue estadísticamente significativa (Tabla 4).

Por lo que se refiere al tipo de actividades realizadas en el Servicio Social, se observa que la atención médica en clínica de tipo rural ha descendido significativamente, con una tendencia clara y marcada, del $83.7 \%$ en 2004 al $61.9 \%$ en 2009 (ji al cuadrado: 128.009; $p<0.0001$ ). 
En cuanto al número de médicos egresados que realizaron alguna especialidad médica, la tendencia es a la baja, mostrando diferencias significativas, es decir, cada vez menos egresados de la carrera hacen alguna especialidad médica; la hicieron el $98.0 \%$ en 2004 y el $80.6 \%$ en 2009 (ji al cuadrado: 62.420; $\mathrm{p}<0.0001)$.

Los promedios porcentuales correspondientes al número de intentos que hicieron los médicos de las seis generaciones para ingresar al sistema de residencias médicas fueron: un intento el $54.3 \%$, dos intentos el $33.5 \%$, tres intentos el $10.3 \%$ y cuatro intentos el $1.4 \%$. La diferencia fue significativa (ji al cuadrado: $77.057 ; p<0.0001$ ).

Se observa que el tiempo en que los médicos lograron su primer empleo ha aumentado. En la generación de 2004 , el $81.6 \%$ lo hizo en menos de 6 meses y en la 2009 solo el 59.7\%; sin embargo, esta diferencia no es significativa (ji al cuadrado: $30.57 ; p<0.203$ ).

\section{Discusión}

Es importante destacar la dificultad para obtener las respuestas de los egresados; como se sabe, en este tipo de investigaciones la tasa es baja ${ }^{18}$. Esta situación se agravó debido a que en la generación de 2004, y en menor importancia en la de 2005, los alumnos no registraban sus correos electrónicos, situación que requirió mayor tiempo y trabajo para lograr las respuestas de los participantes.

El estudio reafirma lo reportado por otros autores acerca de la «feminización» de la carrera de Medicina $^{19}$, encontrando un promedio de un $60 \%$ de mujeres y un $40 \%$ de hombres. Esta feminización se hace evidente si se comparan los resultados con los publicados por Flores Echavarría, et al. ${ }^{20}$, de la Universidad Autónoma Metropolitana en México, correspondientes a los egresados de las generaciones 1989 y 1991, en las que el $64.3 \%$ de sus egresados eran hombres y el $35.7 \%$ mujeres.

Al respecto de los alumnos que trabajaron durante la carrera, se observó un promedio del $14.1 \%$ en las seis generaciones, lo que contrasta con lo encontrado por el mismo grupo de investigadores en los alumnos que concluyeron en 2014 el año de Servicio Social, con un $17.8 \%{ }^{21}$. Lo anterior pareciera indicar que en los últimos años un mayor número de alumnos trabaja y estudia. La Universidad de Rosario en Colombia reportó en 2005 que el $12.8 \%$ de sus alumnos de primer semestre trabajaban en su tiempo libre; de ellos, dos alumnos lo hacían diariamente, lo que relacionaron con reprobación ${ }^{22}$.

El $60 \%$ de los egresados cursó la carrera en 6,5 años sin haber reprobado materias, lo que es consistente con lo reportado por los mismos autores para la generación que terminó el año de Servicio Social en $2014^{20}$.

En el análisis longitudinal de las seis generaciones se pudo observar una tendencia al alza en el número de años para concluir la carrera. En 2004 egresó el $81.6 \%$ en los 6,5 años que marca el programa, mientras que en 2009 solo el $58.7 \%$ lo logró, incrementándose significativamente el número de alumnos que concluyeron en 1 o 2 años más. Cabe destacar que todas las generaciones de estudio correspondieron al mismo Plan Único de Estudios que concluyó en el ciclo 2009-201023.

Otro dato significativo encontrado fue el incremento en el número de materias reprobadas en los alumnos de las últimas tres generaciones. Tanto el incremento en el número de años para cursar la carrera de Medicina como el incremento en el número de materias reprobadas por alumno, principalmente en los ciclos básicos, deben llamar la atención para mejorar la evaluación diagnóstica de ingreso, ya que como reportaron Muñoz, et al. ${ }^{23}$ existe una correlación importante entre esta evaluación y el rendimiento académico de los alumnos, lo que se constituye en una herramienta que puede y debe contribuir a evitar el fracaso escolar.

El perfil profesional de los egresados definido en el Plan Único de Estudios, de la Facultad de Medicina, pretende formar médicos generales de alta calidad, capaces de referir oportunamente a sus pacientes para atención especializada, evitando riesgos innecesarios; efectuar acciones curativas y preventivas; además del trabajo en equipo y la atención integral de los pacientes, considerando los aspectos afectivos y emocionales de los pacientes, siempre bajo estrictas normas éticas y humanas ${ }^{24}$. De acuerdo con las respuestas de los egresados, la mayor parte de ellos consideró haber adquirido estas competencias; sin embargo, un alto porcentaje consideraron no haber obtenido los conocimientos y las habilidades suficientes en lo relacionado con aspectos médico-legales y con la comprensión de estudios epidemiológicos y de investigación socio-médica, lo que genera una gran oportunidad para incidir en el programa de estudios actual y mejorar la formación de las próximas generaciones de médicos. 
En este mismo rubro de aspectos escolares resulta evidente la disminución paulatina en el número de plazas de Servicio Social dedicadas a la atención médica rural, lo cual se debe básicamente a dos factores: el primero, el incremento en el número de escuelas y facultades en el país que hacen autosuficientes a casi todas las entidades federativas en la ocupación de puestos médicos para pasantes en zonas de alta vulnerabilidad; el segundo, las condiciones de inseguridad que prevalecen en diferentes regiones del país y que dificultan la supervisión y la vigilancia por parte de las autoridades y de la propia universidad, tal como reportó Hamui ${ }^{25}$.

Al respecto de la inserción laboral de los egresados, se pudo observar que casi dos terceras partes de las seis generaciones se incorporó a trabajar como médico antes de 6 meses, y un $10.3 \%$ antes de 1 año, lo que constituye información muy relevante, ya que a decir del reporte de la Subsecretaría de Educación Superior, en 2009 , el $78 \%$ de los egresados encontró trabajo antes de 2 años ${ }^{15}$. También se observó que cuatro de cada cinco trabajan en el sector público y uno de cada cinco en lo privado únicamente, lo que corresponde al perfil social de la misión institucional23. Un hecho relevante que se pudo observar en el análisis longitudinal fue que, en la medida que transcurre el tiempo, ha sido más lenta la incorporación de los médicos al mercado de trabajo, y aunque esta variable no tuvo significancia estadística, es notable que en 2004 el $81.6 \%$ de los egresados tenían trabajo antes de 6 meses y para la generación de 2009 solo el $59.7 \%$ lo consiguió en el mismo plazo. No obstante que estos datos pudieran parecer preocupantes, el porcentaje de desempleo general encontrado fue muy bajo (2.9\%), lo que contrasta con otros reportes, como el de Vries, et al. ${ }^{26}$ del $5.7 \%$ o el de Burgos y López ${ }^{15}$, que reportaron en diversas disciplinas, incluyendo la médica, hasta un $14 \%$.

Llama la atención que los médicos de estas generaciones, a pesar de tener relativamente poco tiempo de haber egresado, han dejado de lado la actualización y la capacitación continua, que solo poco más de la mitad (51.9\%) participaron en una a tres actividades de educación médica continua por año, y que el $22 \%$ no han recibido ningún tipo de actualización desde su egreso, lo que se traduce en una gran debilidad en su profesionalismo médico y del propio sistema de salud ${ }^{26}$, pilar fundamental del ejercicio profesional y reto a vencer en la formación de las futuras generaciones.

\section{Conclusiones}

Es importante contar con expedientes completos de los alumnos con el objeto de mejorar el PSAE de la Facultad de Medicina; así mismo, sensibilizar a los alumnos de la importancia de responder a estas encuestas.

Resulta satisfactorio el hecho de que los egresados tienen una excelente tasa de inserción laboral antes de 6 meses, con un nivel de desempleo muy bajo y con un alto grado de satisfacción en su formación y al respecto de su casa de estudios.

Será de gran utilidad investigar lo que está ocurriendo a más profundidad al respecto del incremento en el número de años y de materias reprobadas en las últimas generaciones, además de mejorar la formación de los egresados en las áreas administrativas y de investigación epidemiológica y socio-médica.

También se puede concluir que ninguna de las teorías referidas al inicio de este manuscrito logra explicar el binomio educación vs. trabajo; pareciera que en la actualidad se hacen más evidentes las teorías de la fila, de devaluación de los certificados, del bien psicosocial y del conocimiento heterogéneo.

\section{Agradecimientos}

Agradecemos la valiosa participación en este proyecto del Dr. Alan Barrell, traductor del resumen.

\section{Conflicto de intereses}

Los autores declaran no tener ningún conflicto de intereses.

\section{Responsabilidades éticas}

Protección de personas y animales. Los autores declaran que para esta investigación no se han realizado experimentos en seres humanos ni en animales.

Confidencialidad de los datos. Los autores declaran que han seguido los protocolos de su centro de trabajo sobre la publicación de datos de alumnos y regresados.

Derecho a la privacidad y consentimiento informado. Los autores declaran que en este artículo no aparecen datos de alumnos ni egresados.

\section{Bibliografía}

1. Flores $E$, Sánchez $F$, Coronado $H$, Amador $C$. La formación médica en México y los procesos de búsqueda de garantizar la calidad de los egresados. Rev Fac Med UNAM. 2001;4:195-7. 
2. Glosario de términos usados en el Sistema Nacional de Acreditación Consejo Mexicano para la Acreditación de la Educación Médica (COMAEM), 2008. (Consultado el 27/09/17.) Disponible en: http://www.comaem.org.mx/joomla/images/5.\%20Glosario.pdf

3. UNESCO. Conferencia Mundial sobre la Educación Superior. La educación superior en el siglo XXI. Visión y Acción. Tomo I. Informe Final. París; 1998.

4. Asociación Nacional de Universidades e Instituciones de Educación Superior (ANUIES). Esquema básico para estudios de egresados en Educación Superior. México; 1998.

5. Valenti, G. Varela G. Esquema básico para estudio de egresados: construcción analítica del estudio de egresados. México; ANUIES: 1998.

6. Universidad Veracruzana. Programa de seguimiento de egresados y opinión de empleadores. México: UV: 2009. (Consultado el 27/09/17.) Disponible en: http://www.uv.mx/orizaba/ enfermeria/files/2012/11/ PROGRAMADE SEGUIMIENTO DEEGRESADOSUV.pdf

7. Guerra J, Tapia D, Galicia R, Hashimoto B, Ibarra M, Monje J. Estudio y seguimiento de egresados en la Facultad de Estudios Superiores Iztacala. Un estudio naturalmente multidisciplinario (folleto Folio 3). México: Universidad Nacional Autónoma de México; 2006.

8. Instituto Politécnico Nacional (IPN). Metodología para realizar estudios de seguimiento de egresados en el Instituto Politécnico Nacional. México: IPN; 2007.

9. Alonso C, González L, López M, Azcona V. Indicadores básicos de seguimiento de egresados. Consejo Estatal para el Diálogo con los Sectores Productivos. Jalisco, México; 2011.

10. Romero S, González G. Desempeño de los egresados de medicina en el Examen Nacional de Residencias Médicas: un estudio en la Universidad de Guadalajara. México: Universidad de Guadalajara; 2007.

11. Morales A. El seguimiento de egresados como herramienta para el análisis de la relación entre educación y empleo. Revista Diversidades, Universidad de León. 2011; Sept.-Dic: 21-22.

12. Martínez M. La integración laboral del egresado de educación superior en México. Tec de Monterrey, campus Morelia. 2016. (Consultado el 12/09/17.) Disponible en: https://administradorjorgevelcas.files.wordpress.com/2016/02/folio_008-situacion-lab-egresados.pdf

13. Jara D, Velarde H, Gordillo G, Guerra G, León I, Arroyo C, et al. Factores influyentes en el rendimiento académico de estudiantes del primer año de medicina. An Fac Med. 2008;69:193-7.

14. Universidad Autónoma de Baja California Sur. Programa Institucional de Seguimiento de Egresados. 2012. (Consultado el 12/09/17.) Disponible en: file:///C:/Users/ Miguel\%20Angel/Downloads/ 25042013_110437 PROGRAMA\% 20DE\%20SEGUIMIENTO \%20DE\%20 EGRESADOS.pd
15. Burgos Flores B, López Montes K. La situación del mercado laboral. Revista de la Educación Superior. 2010;39:19-33. (Consultado el 12/09/17.) Disponible en: http://publicaciones. anuies.mx/pdfs/revista/ Revista156 S1A2ES.pdf

16. Ethical Standards of American Educational Research Association. 2011. (Consultado el 19/06/17.) Disponible en: http://www.aera.net/ Portals/38/ docs/Abou t AERA/CodeOfEthics(1).pdf

17. Asociación Médica Mundial. Declaración de Helsinki. 64. a Asamblea General. Fortaleza, Brasil, octubre de 2013. (Consultado el 19/06/17.) Disponible en: file:///C:/ Users/Miguel\%20Angel/ Downloads/Declaraci\% C3\%B3n Helsinki 2013.pdf

18. Aldana G, Morales F, Sabogal F, Ospina A. Seguimiento a egresados. Su importancia para las instituciones de educación superior. Rev Teoría y Praxis Investigativa. 2008;3:61-5.

19. Acosta A, Cortes G, Vélez G, Herrera Z. Seguimiento de egresados de la Facultad de Medicina de la UNAM. Revista de la Educación Superior. 2004;33:7-20

20. Flores Echavarría R, Sánchez Flores A, Coronado Herrera M, Amador Campos JC. La formación médica en México y los procesos en búsqueda de garantizar la calidad de los egresados. Rev Fac Med UNAM. 2001;44:75-80.

21. Fernández-Ortega M, Ortiz-Montalvo A, Ponce-Rosas E, Fajardo-Ortiz G, Mazón-Ramírez J. Caracterización de alumnos de la carrera de Medicina. Rev Inv Ed Med. 2016;5:148-54.

22. Vélez A, Roa CN. Factores asociados al rendimiento académico en estudiantes de medicina. Edu Med. 2005;8:74-82.

23. Muñoz-Comonfort A, Leenen I, Fortul-van der Goes T. Correlación entre la evaluación diagnóstica y el rendimiento académico de los estudiantes de Medicina. Inv Ed Med. 2014;3:85-91.

24. Facultad de Medicina. Plan Único de Estudios de la Carrera de Médico Cirujano 2009. Facultad de Medicina, UNAM. (Consultado el 12/09/17.) Disponible en: http://www.facmed.unam. mx/plan/unico/index.pdf

25. Hamui Sutton A. Condiciones sociales en que los pasantes de la Facultad de Medicina de la Universidad Nacional Autónoma de México realizan el servicio social en áreas rurales. Gac Med Mex. 2012;148:277-80.

26. Vries W, Navarro Y. ¿Profesionales del futuro o futuros taxistas? Los egresados universitarios y el mercado laboral en México. Rev lberoamericana de Educación Superior. 2011;2:3-27. (Consultado el 12/09/17.) Disponible en: https://ries. universia.net/article/ view/51/-profesionistas-futuro- futuros-taxistas-egresados- universitarios-mercado- laboral-mexico 